\title{
Prevalence and Risk Factors of Metabolic Syndrome in Pregnant Women in the Centre and Littoral Regions of Cameroon
}

\author{
Jules Destin Djeufouata ${ }^{1}$, Walter Ebot Ojong ${ }^{1}$, Theophile Nana Njamen ${ }^{2}$, \\ Jules Clement Nguedia Assob ${ }^{1,3}$, Bruno Phelix Telefo, * \\ ${ }^{1}$ Department of Medical Laboratory Sciences, Faculty of Health Sciences, University of Buea, Buea, Cameroon \\ ${ }^{2}$ Department of Obstetrics and Gynecology, Faculty of Health Sciences, University of Buea, Buea, Cameroon \\ ${ }^{3}$ Department of Biomedical Sciences, Faculty of Medicine and Pharmaceutical Sciences, University of Douala, Douala, Cameroon \\ ${ }^{4}$ Department of Biomedical Sciences, Faculty of Medicine and Biomedical Sciences, University of Dschang, Dschang, Cameroon
}

Email address:

Julesdestin00@gmail.com (J. D. Djeufouata), ebotwally@yahoo.com (W. E. Ojong), njanatheo@yahoo.fr (T. N. Njamen), juleclement@yahoo.fr (J. C. N. Assob), bphelix@yahoo.co.uk (B. P. Telefo)

${ }^{*}$ Corresponding author

\section{To cite this article:}

Jules Destin Djeufouata, Walter Ebot Ojong, Theophile Nana Njamen, Jules Clement Nguedia Assob, Bruno Phelix Telefo. Prevalence and Risk Factors of Metabolic Syndrome in Pregnant Women in the Centre and Littoral Regions of Cameroon. European Journal of Clinical and Biomedical Sciences. Vol. 6, No. 5, 2020, pp. 104-115. doi: 10.11648/j.ejcbs.20200605.16

Received: June 21, 2020; Accepted: July 9, 2020; Published: October 13, 2020

\begin{abstract}
Background: The prevalence of metabolic syndrome (MS) has increased exponentially this last decades in sub Saharan Africa. The aim of this study was to determine the prevalence of metabolic syndrome, its components and the risk factors associated with metabolic syndrome in Cameroonian pregnant women. Methods: It was a hospital-based cross sectional study involving pregnant women recruited in 16 public health facilities in the Centre and Littoral Regions of Cameroon. Sociodemographic factors and medical history were recorded using a structured questionnaire. Blood samples were collected and biochemical analyses were performed at the Laboratory Unit of the Bangangté District Hospital. The criteria given by National Cholesterol Education Program Adult Treatment Panel III (NCEP ATP III 2004) were modified to adapt in pregnancy state and used for assessment of metabolic syndrome. The Chi-square test, Pearson correlation test, Student-test, and multivariable logistic regression were used in this study. Results: A total of 859 pregnant women aged from 17 to 45 years were enrolled. The prevalence of metabolic syndrome was $7.0 \%$. The prevalence of individual components of metabolic syndrome were: hyperglycaemia (47.1\%), obesity (24.0\%), hypertension (20.5\%), Low High Density Lipoprotein Cholesterol (16.3\%) and hypertriglyceridaemia (3.7\%). Participants with metabolic syndrome had higher mean values of systolic blood pressure, diastolic blood pressure, fasting blood glucose, body mass index, triglycerides and lower high density lipoprotein cholesterol compared to those without metabolic syndrome. There was no significant association between socio-demographic factors and metabolic syndrome. High parity, family (parents) history of type 2 diabetes mellitus were positively associated with metabolic syndrome. Parity and gravidity were positively correlated with obesity. Obesity, hyperglycaemia, high systolic blood pressure, low high density lipoprotein cholesterol were significantly associated with metabolic syndrome. Furthermore, $72.6 \%$ of participants displayed at least one risk factor of metabolic syndrome. Conclusion: Metabolic syndrome is common in the Cameroonian pregnant women and its most prevalent components are hyperglycaemia and obesity. Increased in parity and parent history of type 2 diabetes mellitus were significantly associated with metabolic syndrome. Also, more than two-third of participants have at least one component of metabolic syndrome. The high prevalence of metabolic syndrome, hyperglycemia, obesity and hypertension demonstrates the need to closely follow up pregnant women in order to minimize the risk of metabolic syndrome, cardiovascular diseases, type 2 diabetes mellitus, maternal and foetal adverse outcomes.
\end{abstract}

Keywords: Metabolic Syndrome, Prevalence, Pregnancy, Metabolic Syndrome Components, Association, Risk Factors 


\section{Introduction}

Metabolic syndrome (MS) is a cluster of several risk factors including high fasting blood glucose, high blood pressure, elevated triglycerides levels, low high-density lipoprotein cholesterol levels, and obesity that predisposes individuals to a high risk of cardiovascular diseases (CVD) and type 2 diabetes mellitus (T2DM) [1,2].

The prevalence of metabolic syndrome has been increasing in the last decade worldwide and is becoming a public health concern nowadays [3]. The worldwide prevalence of metabolic syndrome in the general population range from 10 to $84 \%$, depending on the region, the population studied and the diagnostic criteria used $[4,5]$. The prevalence of metabolic syndrome and its components are also influenced by sociodemographic factors, economic status, lifestyle, diet, obstetrical and medical history, genetic background and ethnicity $[6,7]$.

In Cameroon, the prevalence of metabolic syndrome is reported to be $7 \%$ in rural areas, $17.4 \%$ in less urbanized areas and $12.7 \%$ in urban areas using International Diabetes Federation (IDF) definition [8]. A study conducted by dos Prazeres and collaborators [9] on Angolan pregnant women found the prevalence of metabolic syndrome to be as high as 29.2\% based on the National Cholesterol Education Program Adult Treatment Panel III criteria.

Pregnancy is a timed physiological condition, however, there is some evidence that this condition may have a long term impact on women's health; it is generally assumed that pregnancy associated insulin resistance resolves after delivery, but subtle metabolic changes could persist $[10,11]$. Pregnancy is known as a period of weight gain, hyperlipidaemia and insulin resistance, thus it is a critical period in which the components of metabolic syndrome can set in rapidly alongside with adverse effects on the maternal and fetal outcomes [12].

As risk factors of metabolic syndrome, sometimes considered as tomorrow's diseases, are often preventable, appropriate health intervention before, during and after pregnancy can significantly reduce their prevalence as well as adverse maternal and fetal outcomes.

To the best of our knowledge, there are scarce published data on the prevalence of metabolic syndrome in pregnancy in Africa and none in Cameroon. The aim of the present study was to determine the prevalence of metabolic syndrome, and its individual components among pregnant women in Cameroon, and the factors associated with metabolic syndrome in the study population.

\section{Materials and Methods}

\subsection{Framework, Type and Period of Study}

It was a hospital-based cross-sectional, descriptive and analytical study carried out in sixteen health facilities in the Centre and Littoral Regions of Cameroon from June $1^{\text {st }}, 2018$ to August $2^{\text {nd }}, 2019$.

Socio-demographic and anthropometric data were collected using a structured questionnaire. Biochemical analyses were carried out at the Laboratory Unit of Bangangté District Hospital.

\subsection{Ethical Considerations}

The experimental protocol was approved by the Centre Regional Ethics Committee for Human Health Research (CRERSH/C) CE No 1545/CREESHC/18. Administrative authorizations were obtained from the Regional Delegate of Public Heath for Centre and Littoral Regions, from the Directors of District Hospitals of different study sites as well as from the Faculty of Health Sciences authorities. All stages of the research were carried out in strict compliance with the rules set out in the Helsinki Declaration.

\subsection{Study Population}

Apparently healthy pregnant women aged 17 to 45 years, born of two black Cameroonian parents, permanently resident in the data collection areas for at least one year who signed the informed consent form were included in the study.

Participants who did not fulfill the criteria above and or who are physically handicapped, were mentally ill, had uncontrolled endocrine disease, renal failure, complicated pregnancy were excluded from the study.

Adolescents less than 17 and women over 45 years of age were excluded because these may lead to high-risk pregnancy.

\subsection{Sampling Method and Sample Size}

We applied the multi-stage, proportionate sampling strategy for the various study sites and consecutive proportionate sampling for the selection of participants.

The sample size was obtained using the LORENZ formula $\left(t^{2} p(1-p) / e^{2}\right)$. Since the prevalence of metabolic syndrome in Cameroonian pregnant women is unknown, we assumed a prevalence (p) of $50 \%$. With a minimum confidence level of $95 \%$ and maximum likely error (e) of $5 \% . \mathrm{t}=\%$ point corresponding to significant level of $95 \%=1.96$.

The sampling design and value of imponderable (no answers, registration errors...) were set at 2 and $10 \%$ respectively.

The calculated sample size was therefore 847 participants.

\subsection{Data Collection}

Data were collected by four laboratory technicians using structured a questionnaire divided into four sections, (1) socio-demographic characteristics, (2) eating habits, lifestyle and medical history, (3) anthropometric and physiological measurements and (4) biochemical evaluation.

\subsubsection{Administration of Questionnaires}

The questionnaire was designed according to modified model "WHO STEPS instrument for chronic diseases" [13], written in English and French and administered in a step by step and face to face approach. This was preceded by the 
signature of the informed consent.

\subsubsection{Anthropometric Data}

Participants anthropometric data were measured with lightly clad, without jackets/coats, head coverings, hair clips or shoes and with empty pockets. The following measurements were taken and recorded.

Weight was measured using a calibrated mechanical scales (SECA Gmbh and Co, Germany) to the nearest $0.1 \mathrm{~kg}$ with maximum capacity of $200 \mathrm{~kg}$. Pre-pregnancy weight was self-reported by participants or by investigators if it was available from hospital book or medical record.

The height was measured with participant, in a vertical position, using a wall-mounted Stadiometer and was recorded at the nearest centimeters.

Body Mass Index (BMI) was calculated using Quételet's

$$
\text { formula: } B M I=\frac{m}{H^{2}}
$$

with: $\mathrm{m}=$ mass of subject in kilograms, $\mathrm{H}^{2}=$ Square of height (in $\mathrm{m}^{2}$ ).

\subsubsection{Measurement of Arterial Blood Pressure}

Arterial blood pressure was measured by trained nurses and the principal investigator while the participant was seated for 10 minutes with an appropriately sized cuff using a functional, calibrated blood pressure monitor Omron ${ }^{\circledR}$ HEM7124. Readings were taken before blood was drawn for biochemical analyses. Three readings were taken and an average value for the blood pressure recorded.

\subsubsection{Determination of Biological Parameters}

a) Sample collection and preparation:

A total of $10 \mathrm{~mL}$ of blood was collected by venous phlebotomy into a dry vacutainer ${ }^{\circledR}$ tube from each participant after an overnight fast of at least 12 hours for biochemical analysis. These samples were then centrifuged for 15 minutes at $3000 \mathrm{rpm}$ within one hour of blood collection. Then, sera was separated into two Ependorf tubes, labeled and kept at a $-20^{\circ} \mathrm{C}$ in a freezer until the tests were performed.

The serum obtained was used for the determination of lipid profile on Urit $\AA-810$ Spectrophotometer (Guilin-Guangxi, China) within 5 days after blood collection.

Capillary blood sample was also taken using finger prick with a sterile lancet after cleaning the site with antiseptic alcohol swaps for glucose measurement.

b) Determination of lipid profile

Serum total cholesterol (TC) was determined by colorimetric CHOD-PAP method with Advanced Turbidity Clearing System (ATCS) [14], using Dialab ${ }^{\circledR}$ reagent kits, Wiener Neudorf, Austria.

Serum triglycerides (TG) concentration was analyzed at $546 \mathrm{~nm}$ by the GPO-PAP (Glycerol-3-Phosphate Oxydase Peroxidase-4-Aminoantipyrin) Trinder method [15] using Chronolab $^{\circledR}$ (Chronolab ${ }^{\circledR}$ Barcelona, Spain) reagent kits.

Serum High Density Lipoprotein Cholesterol was analyzed by colorimetric, phosphotugstic acid method [16] using Dialab $^{\circledR}$ reagent kits, Wiener Neudorf, Austria.

The low density lipoprotein cholesterol (LDL-C) level was determined using the Friedewald formula [17]: For the concentration in $\mathrm{g} / \mathrm{L}:[L D L-C]=[T C]-([H D L-C]+[T G] / 5)$

This formula is applicable if TG level is less than $3.5 \mathrm{~g} / \mathrm{l}$.

c) Determination of fasting blood glucose

Fasting capillary blood glucose of each participant was measured with a validated One Touch Ultra ${ }^{\circledR}$ monitoring device.

All specimens and reagents were handled according to the manufacturer instructions.

\subsection{Data Entry and Analysis}

After the manual counting of survey forms and biological analysis, the data were coded, entered into a computer using Epi-data $^{\circledR}$, Microsoft Excel 2007 for windows XP and finally transferred into the Statistical Package for Social Sciences (SPSS, version 19) for data analysis.

The results were summarized by the use of descriptive statistics. Continuous variables were reported as means \pm standard deviations (SD) and categorical variables as percentages.

The chi-square $\left(\chi^{2}\right)$ test was used to establish the associations between the categorical variables and the student test (t-test) for the comparison of means in the different subgroups of the study population.

Pearson correlation test was used to determine the association between continuous variables.

Logistic regression analysis was used to assess the relationships between different variables and metabolic syndrome.

The results were considered statistically significant at $\mathrm{p}$ value $<0.05$

\subsection{Definition of Variables}

As there is no approved criterion of metabolic syndrome in the obstetric population, to take into account the modification in anatomy and lipid profile which occurs in normal pregnancy, we used the modified the NCEP ATP III definition criteria by relying on the definition of dyslipidaemia in pregnancy according to Piechota and Staszekski [18] percentiles criteria. Also obesity was defined using pre-pregnancy BMI or BMI before the 13 weeks of pregnancy.

Metabolic syndrome and its individual components were defined according to the modified criteria of NCEP ATP III as the presence of three or more of any of the following components: serum triglycerides levels $>1.75 \mathrm{~g} / \mathrm{L}$ at the $1^{\text {st }}$ quarter of pregnancy, $>2.54 \mathrm{~g} / \mathrm{L}$ at $2^{\text {nd }}$ quarter, at $3^{\text {rd }}$ quarter $>$ $4.14 \mathrm{~g} / \mathrm{L}$ or use of low triglycerides drugs; HDL-C $<0.35 \mathrm{~g} / \mathrm{L}$ at $1^{\text {st }}$ quarter of pregnancy, $2^{\text {nd }}$ quarter $<0.42 \mathrm{~g} / \mathrm{L}, 3^{\text {rd }}$ quarter $<0.40 \mathrm{~g} / \mathrm{L}$, arterial blood pressure (Systolic blood pressure/ diastolic blood pressure) $\geq 130 / 85 \mathrm{mmHg}$ or use of antihypertensive drugs, fasting blood glucose (FBG) $\geq 1.01 \mathrm{~g} / \mathrm{L}$ or use of diabetes drugs; pre pregnancy BMI or BMI before 13 weeks of pregnancy $\geq 30 \mathrm{Kg} / \mathrm{m}^{2}$ ). 
Socio-demographic characteristics included educational levels, occupation, ethnic group, marital, religious, economic status and age.

Participants were grouped into the Bantu, Semi-Bantu and Sudanese ethnic groups.

Economic status was classified into poor (expense $<1.56$ United State Dollar (USD) a day), moderate (expense $\geq 1.56$ USD a day) and good.

Education level was grouped into: no formal, primary, secondary, and higher education.

Marital status was categorized into two groups: married/cohabiting and single.

Medical history in the present study included parity, gravidity, family (parent's) history of hypertension, type 2 diabetes mellitus and obesity.

Parity was defined as the number of deliveries beyond 24 weeks of gestation regardless of whether the child was born alive or was stillborn while gravidity was defined as the number of pregnancies, including lost pregnancies due to stillbirths or miscarriages.

Hypertension was defined as systolic / diastolic blood pressure $(\mathrm{SBP} / \mathrm{DBP}) \geq 130 / 85 \mathrm{mmHg}$.

Obesity was defined as BMI or pre-pregnancy BMI $\geq 30.0$ $\left.\mathrm{kg} / \mathrm{m}^{2}\right)$.

\section{Results}

\subsection{Socio-Demographic Characteristic of the Study Population}

The study was carried out in sixteen public health facilities in the Centre Region and the Littoral Regions of Cameroon.

Out of 960 pregnant women recruited in the two regions, 859 were included in the study and 101 excluded from the study.

Table 1 indicates that most of participants were married or cohabiting, Christians, had attended secondary school and had moderate income.

Table 1. Socio-Demographic and Economic Characteristics of the Study Population.

\begin{tabular}{|c|c|c|c|}
\hline Characteristics & & N (Sample size) & Percentage (\%) \\
\hline \multirow{4}{*}{ Level of study } & No formal education & 01 & 0.1 \\
\hline & Primary & 63 & 7.3 \\
\hline & Secondary & 549 & 63.9 \\
\hline & Higher & 246 & 28.7 \\
\hline \multirow{3}{*}{ Marital status } & Single & 294 & 34.2 \\
\hline & Married/cohabiting & 564 & 65.7 \\
\hline & Divorced & 01 & 0.1 \\
\hline \multirow{3}{*}{ Family economic status } & Poor $<1.56$ USD / day & 91 & 10.6 \\
\hline & Moderate & 745 & 86.7 \\
\hline & Good & 23 & 2.7 \\
\hline \multirow{3}{*}{ Occupation } & Employed & 117 & 13.6 \\
\hline & Non-employed & 498 & 58.0 \\
\hline & Business & 244 & 28.4 \\
\hline \multirow{3}{*}{ Ethnic group } & Bantu & 413 & 48.1 \\
\hline & Semi -Bantu & 409 & 47.6 \\
\hline & Sudanese & 37 & 4.3 \\
\hline \multirow{4}{*}{ Religion group } & Catholic & 536 & 62.4 \\
\hline & Protestant & 245 & 28.5 \\
\hline & Muslim & 34 & 4.0 \\
\hline & Animist /polytheist & 44 & 5.1 \\
\hline \multirow{5}{*}{ Age groups (years) } & $17-22$ & 182 & 21.2 \\
\hline & $23-28$ & 330 & 38.4 \\
\hline & $29-34$ & 263 & 30.6 \\
\hline & $35-40$ & 75 & 8.7 \\
\hline & $41-45$ & 9 & 1.1 \\
\hline Total & & 859 & 100 \\
\hline
\end{tabular}

\subsection{Prevalence of Metabolic Syndrome and Its Components in the Study Population}

The prevalence of metabolic syndrome was $7.0 \%$.

Figure 1 indicates that the most prevalent component of metabolic syndrome was hyperglycaemia, followed by obesity. 


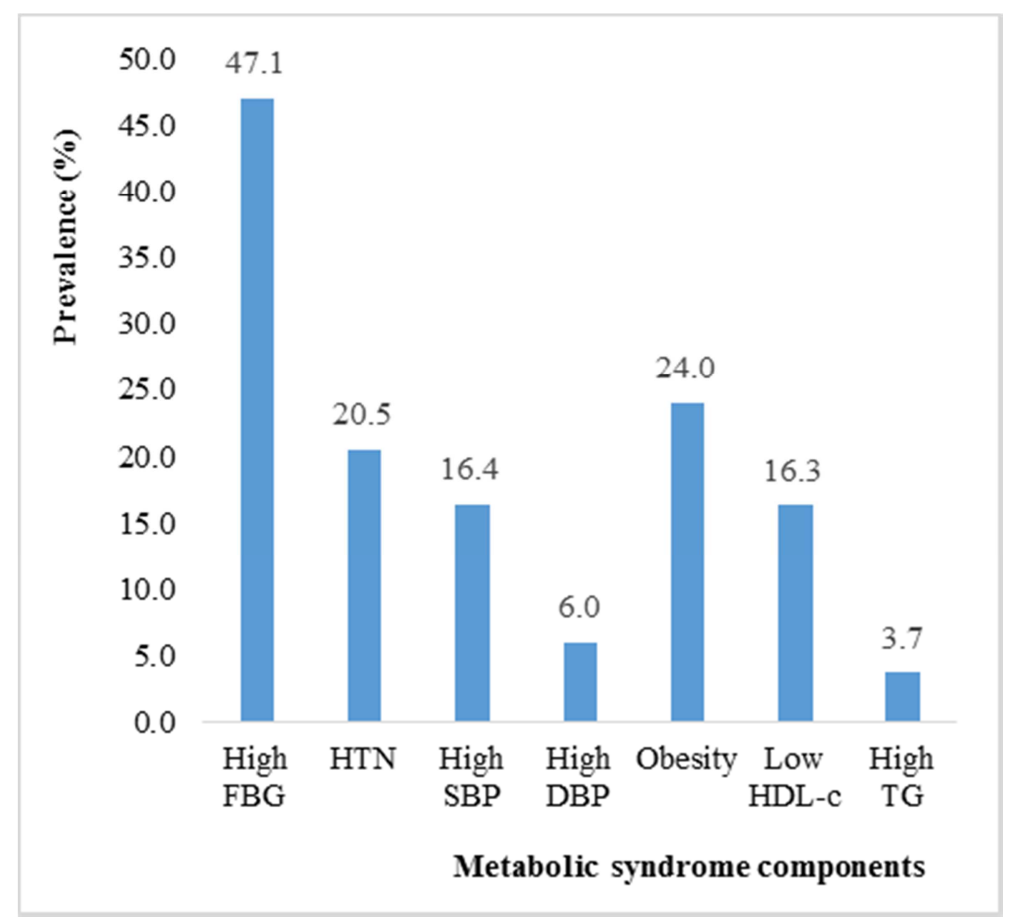

Figure 1. Prevalence of Metabolic Syndrome Components.

FBS: fasting blood sugar, HTN: hypertension, SBP: systolic blood pressure, DBP: diastolic blood pressure, HDL-C : high density lipoprotein cholesterol, TG: triglycerides

Figure 2 reveals that $72.6 \%$ of participants displayed at least one risk factor of metabolic syndrome.

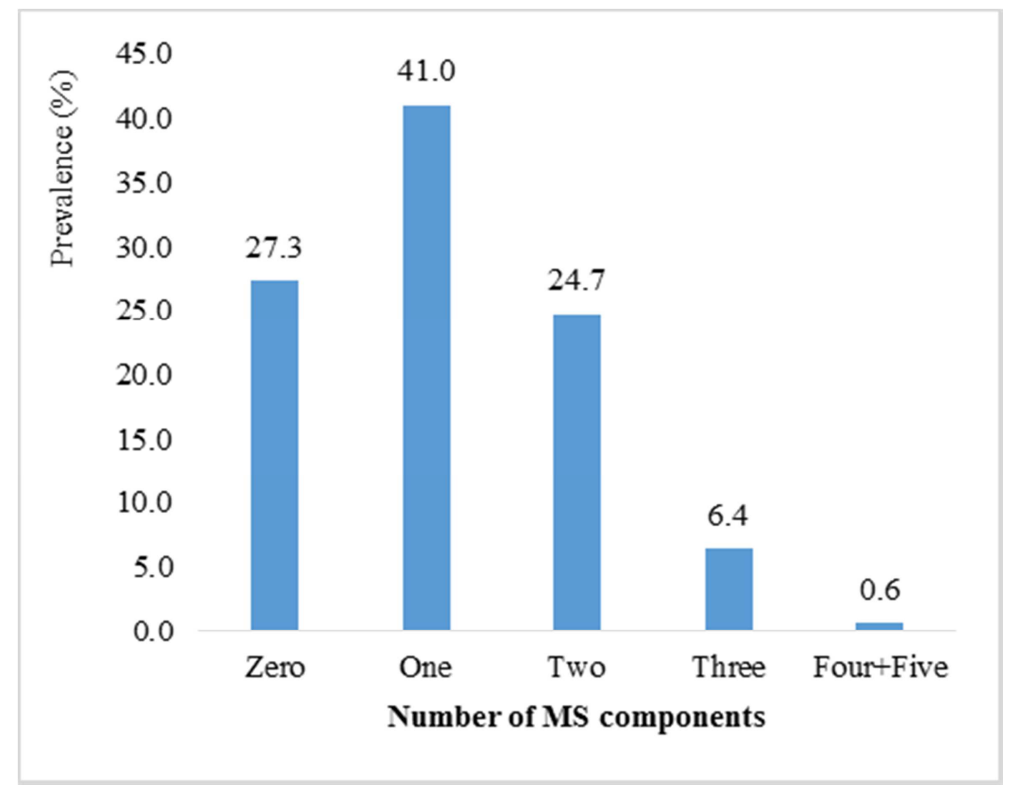

Figure 2. Distribution of Participants According the Number of Metabolic Syndrome's Components.

\subsection{Comparison of Parameters of Participants with and Without Metabolic Syndrome}

Table 2 indicates that participants with metabolic syndrome had significantly higher mean values of serum triglycerides, fasting blood glucose, body mass index, systolic and diastolic blood pressure than those without metabolic syndrome. However, the mean serum HDL-C level was lower in participants with metabolic syndrome than those without it. 
Table 2. Comparison of Physiological and Biochemical Parameters of Participants with and without Metabolic Syndrome.

\begin{tabular}{lllll}
\hline \multirow{2}{*}{ Parameters } & \multirow{2}{*}{ All } & Metabolic syndrome & No & p \\
\cline { 3 - 5 } & & 659 & Yes & 799 \\
N (Sample size) & $27.3 \pm 5.6$ & $29.4 \pm 6.3$ & $27.1 \pm 5.5$ & 0.002 \\
Age (years) & $23.2 \pm 8.0$ & $21.3 \pm 8.6$ & $23.4 \pm 8.0$ & 0.059 \\
Gestational age (weeks) & $2.09 \pm 0.32$ & $2.06 \pm 0.24$ & $2.09 \pm 0.33$ & 0.319 \\
Total cholesterol $(\mathrm{g} / \mathrm{L})$ & $1.29 \pm 0.57$ & $1.49 \pm 0.71$ & $1.28 \pm 0.56$ & 0.005 \\
Triglycerides $(\mathrm{g} / \mathrm{L})$ & $0.56 \pm 0.20$ & $0.46 \pm 0.19$ & $0.58 \pm 0.20$ & 0.000 \\
High density lipoprotein-Cholesterol $(\mathrm{g} / \mathrm{L})$ & $1.26 \pm 0.33$ & $1.29 \pm 0.28$ & $1.26 \pm 0.33$ & 0.455 \\
Low density lipoprotein Cholesterol $(\mathrm{g} / \mathrm{L})$ & $0.99 \pm 0.16$ & $1.12 \pm 0.18$ & $0.99 \pm 0.16$ & 0.000 \\
Fasting blood glucose $(\mathrm{g} / \mathrm{L})$ & $27.08 \pm 4.48$ & $32.15 \pm 5.92$ & $26.67 \pm 4.11$ & 0.000 \\
Body mass index $\left(\mathrm{kg} / \mathrm{m}^{2}\right)$ & $116.9 \pm 12.1$ & $126.8 \pm 13.4$ & $115.8 \pm 11.7$ & 0.000 \\
Systolic blood pressure $(\mathrm{mmHg})$ & $69.5 \pm 10.9$ & $76.4 \pm 13.4$ & $69.0 \pm 10.6$ & 0.000 \\
Diastolic blood pressure $(\mathrm{mmHg})$ & & & \\
\hline
\end{tabular}

Values are expressed as mean \pm standard deviation, $\mathrm{p}$ significant if $<0.05$.

\subsection{Association Between Socio-Demographic Factors and Metabolic Syndrome}

Table 3 shows that there was no significant association between socio-demographic factors and metabolic syndrome.

Table 3. Association of Metabolic Syndrome to Socio-demographic Characteristics.

\begin{tabular}{|c|c|c|c|c|}
\hline & \multicolumn{4}{|c|}{ Metabolic syndrome } \\
\hline & Yes & No & $\chi^{2}$ & $\mathbf{p}$ \\
\hline Educational level & & & 2.35 & 0.308 \\
\hline No formal education -primary & 05 & 58 & & \\
\hline Secondary & 43 & 507 & & \\
\hline Higher /university & 12 & 234 & & \\
\hline Occupation & & & 0.527 & 0.768 \\
\hline Employed & 10 & 107 & & \\
\hline Unemployed & 34 & 464 & & \\
\hline Business & 16 & 228 & & \\
\hline Ethnic group & & & 0.536 & 0.464 \\
\hline Bantu & 32 & 387 & & \\
\hline Semi-Bantu/ Sudanese & 28 & 412 & & \\
\hline Marital status & & & 2.49 & 0.114 \\
\hline Married/cohabitating & 45 & 519 & & \\
\hline Single & 15 & 280 & & \\
\hline Economic status & & & 0.79 & 0.373 \\
\hline Poor $(<1.56$ USD/day $)$ & 08 & 72 & & \\
\hline Moderate and Good (>1.56 USD/day) & 52 & 667 & & \\
\hline Religious status & & & 2.10 & 0.349 \\
\hline Catholics & 42 & 494 & & \\
\hline Protestants & 12 & 229 & & \\
\hline Muslins/ animists/polytheist & 6 & 76 & & \\
\hline Age (years) & & & 0.881 & 0.348 \\
\hline $17-27$ & 28 & 423 & & \\
\hline $28-45$ & 32 & 376 & & \\
\hline
\end{tabular}

$\chi 2=$ chi-square test; USD=United States Dollar, $\mathrm{p}=\mathrm{p}$-value.

\subsection{Association Between Metabolic Syndrome and Its Individual Components}

Observation of a binary logistic regression model with HDL-C, TG, FBG, BMI, SBP and DBP as independent, continuous variables and metabolic syndrome as dependent variable indicates that fasting blood glucose and body mass index predicted most the risk of metabolic syndrome in the study population than other components. Moreover, triglycerides and diastolic blood pressure showed no significant association with metabolic syndrome (table 4).
Table 4. Logistic Regression between Metabolic Syndrome and its Components.

\begin{tabular}{llll}
\hline Parameters & Odd ratio & $\mathbf{9 5 \%} \mathbf{C I}$ & $\mathbf{p}$ \\
\hline HDL-C & 0.000 & $0.00-0.03$ & 0.000 \\
TG & 1.32 & $0.81-2.14$ & 0.263 \\
FBG & 75.67 & $13.52-423.61$ & 0.000 \\
BMI & 1.24 & $1.16-1.33$ & 0.000 \\
SBP & 1.07 & $1.03-1.10$ & 0.000 \\
DBP & 1.02 & $0.99-1.06$ & 0.229 \\
\hline
\end{tabular}

HDL-C: High density lipoprotein cholesterol, TG: triglycerides, FBG: Fasting blood glucose, BMI: Body mass index, SBP: Systolic blood pressure, DBP: Diastolic blood pressure, CI: confidence interval. 


\subsection{Association Between Medical History and Metabolic Syndrome}

\subsubsection{Correlation Between Medical History and Components of Metabolic Syndrome}

Table 5 shows a fair, significant and positive correlation between gravidity and body mass index $(\mathrm{r}=0.22 ; \mathrm{p}<0.001)$. Also parity number was positively and significantly correlated to diastolic blood pressure $(\mathrm{r}=0.09 ; \mathrm{p} \approx 0.008)$ and body mass index $(\mathrm{r}=0.22 ; \mathrm{p}<0.001)$.

Table 5. Correlation between Parity Number, Gravidity Number and Components of Metabolic Syndrome.

\begin{tabular}{lllll}
\hline Components of & \multicolumn{2}{c}{ Gravidity } & \multicolumn{3}{l}{ Parity } \\
\cline { 2 - 5 } MS & $\mathbf{r}$ & $\mathbf{p}$ & $\mathbf{r}$ & $\mathbf{p}$ \\
\hline SBP & -0.06 & 0.100 & -0.03 & 0.380 \\
DBP & 0.05 & 0.115 & 0.09 & 0.008 \\
BMI & 0.22 & 0.000 & 0.22 & 0.000 \\
FBG & 0.02 & 0.589 & -0.01 & 0.722 \\
TG & -0.05 & 0.181 & -0.03 & 0.309 \\
HDL-C & 0.05 & 0.116 & 0.05 & 0.170 \\
\hline
\end{tabular}

$\mathrm{r}=$ correlation coefficient of Pearson; $\mathrm{p}=\mathrm{p}$-value, FBG: Fasting blood glucose, BMI: Body mass index, SBP: Systolic blood pressure, DBP: Diastolic blood pressure.

\subsubsection{Logistic Regression Analysis Between Medical History and Metabolic Syndrome}

Table 6 shows a logistic regression analysis between medical history and metabolic syndrome. Dependent variable was metabolic syndrome (qualitative) while independent variables were parity (quantitative), gravidity (quantitative), history of hypertension (qualitative), history of type 2 diabetes mellitus (qualitative) and history of obesity (qualitative).

Participants with no family history of type 2 diabetes mellitus had a 0.46 fold reduction in the risk of metabolic syndrome compared those with family history of type 2 diabetes mellitus.

Family history of obesity was not significantly associated with metabolic syndrome even after adjusting for gravidity and parent history of hypertension.

Parent's history of hypertension was not significantly associated with metabolic syndrome in the present study.

Each parity increased about a 1.63 fold the risk of developing metabolic syndrome. However, there was no statistically significant association between metabolic syndrome and gravidity.

Table 6. Logistic Regression Analysis between Medical History and Metabolic Syndrome.

\begin{tabular}{|c|c|c|c|c|c|c|}
\hline & \multicolumn{2}{|l|}{ Model I } & \multicolumn{2}{|l|}{ Model II } & \multicolumn{2}{|l|}{ Model III } \\
\hline & OR $(95 \%$ CI $)$ & $\mathbf{p}$ & OR $(95 \%$ CI $)$ & $\mathbf{p}$ & OR $(95 \% \mathrm{CI})$ & $\mathbf{p}$ \\
\hline Gravidity & $1.03(0.59-1.79)$ & 0.917 & - & - & - & - \\
\hline Parity & $1.59(0.91-2.80)$ & 0.106 & $1.63(1.11-2.40)$ & 0.013 & $1.63(1.11-2.40)$ & 0.013 \\
\hline History of hypertension & $1.04(0.57-0.19)$ & 0.894 & $1.04(0.57-1.89)$ & 0.899 & - & - \\
\hline History of type 2 diabetes mellitus & $0.46(0.26-0.81)$ & 0.007 & $0.46(0.26-0.81)$ & 0.007 & $0.46(0.27-0.79)$ & 0.006 \\
\hline History of obesity & $0.69(0.39-1.22)$ & 0.208 & $0.69(0.39-1.22)$ & 0.206 & $0.69(0.39-1.22)$ & 0.206 \\
\hline
\end{tabular}

Model I: crude model, Model II: adjusted for gravidity, Model III: adjusted for gravidity and history of hypertension.

OR: odd ratio, $95 \% \mathrm{CI}$ : confidence interval.

\section{Discussion}

\subsection{Socio-Demographic Characteristic of the Study Population}

The study participants were relatively young. This could be justified by the fact that participants below 17 years above 45 years were excluded from the study.

The present study showed that $92.5 \%$ of participants were holders of at least First School Leaving Certificate and only one participant was not educated. This is similar to educational level reported by Halle et al [19]. Also, these finding is consistent with the high literacy level of $64.8 \%$ among female Cameroonians [20].

Most of participants $(65.7 \%)$ were living as couples (married or cohabiting) while $34.3 \%$ were single women. This could be explained by their age and the level of education (50\% of participants were above 27 years and were educated).

A majority of participants (58\%) were unemployed. This could be justified by their age and sex. Indeed, our study population consisted of pregnant women with age ranging from 17 to 45 years old with a median age of 27 years, this means that $50 \%$ of them had age ranging from 17 to 27 years old; they were of school age. Moreover, unpaid housework takes up most of women's time compared to men; and unemployement is known to increase with education level [21].

The common major ethnic groups found in the present study were Bantu (48.1\%) and Semi-Bantu (47.6\%). This contrasts the national distribution of the population according to ethnicity which is Semi-bantu (38\%), Bantu (30\%) and Sudanese (32\%) [22]. This difference could be explained by the fact that Northern Cameroon is occupied mainly by Sudanese while the Southern Cameroon is populated mostly by Bantu and Semi-Bantu ethnic groups

The distribution of participants according to religion is in line with Cameroon 2005 census report, which found that $69.2 \%$ of population was Christian, $20.9 \%$ was Muslims and $9.9 \%$ animists or others religions [23].

Majority of participants in the present study had moderate economic status. This contrast with report of ECAM 4 [20] which found that $37.5 \%$ of Cameroonians live on 1.56 USD a day. This inconsistency could be explained by the fact that most participants were residents of Douala and Yaoundé, two urban areas Littoral and Centre Regions of Cameroon. Thus, 
estimating poverty using the baseline of dally income of 1.56 USD would underestimate the level of poverty and therefore the result obtained in this study. Also, we did not take into consideration the number of person living in the same house with no financial income.

\subsection{Prevalence of Metabolic Syndrome and Its Components}

The high prevalence of metabolic syndrome found in the present study could be explained by a higher prevalence of hyperglycaemia, obesity and hypertension found in the present study. Similarly, this prevalence is higher than 3\% reported by Lima et al. [24] in 200 pregnant women before 16 weeks of pregnancy in the basic health units of the city of Campina Grande (Brazil); this difference, however may have resulted from the application of different criteria of metabolic syndrome and the size of the study population. On the contrary, the prevalence of metabolic syndrome found in the present study $(7.0 \%$ ) was lower than $29.2 \%$ reported by dos Prazeres et al [9] in the study in the prevalence of metabolic syndrome in non-diabetic Angolan pregnant women. This difference could be justified by the modification of metabolic syndrome's definition used in both studies. In the Angolan study, waist circumference was used to define obesity while in the present study pre-pregnancy BMI or BMI in the first quarter of pregnancy was used, this overestimated the rate of obesity in their study as pregnancy is characterized by the progressive and profound increase of abdominal circumference, with a consequence on the rate of metabolic syndrome. Moreover, this difference could be justified by differences of the socio-demographic, lifestyle and genetic characteristics of the two populations.

The prevalence of obesity in the present study (24\%) is almost similar to $19.5 \%$ reported by Kamadjeu et al [25] among women in 4 urban areas in Cameroon. However, this prevalence is higher than $11.1 \%$ reported by dos Prazeres et al. [9] in non-diabetic Angolan pregnant women. The reasons could be that, dos Prazeres et al [9] defined obesity as prepregnancy body mass index above $30 \mathrm{~kg} / \mathrm{m}^{2}$ while in the present study obesity was defined as pre-BMI or BMI before 13 weeks of pregnancy $\geq 30 \mathrm{~kg} / \mathrm{m}^{2}$, which would overestimate the prevalence of obesity.

The overall prevalence of hypertension in the present study is lower than the Cameroon national average of $31.0 \%$ [26]. This difference could be due to the characteristic of the study participants. In the present study, participants consisted of pregnant women while in the national campaign it is the general population that was considered. In fact, women are much more protected against hypertension before menopause due to ovarian hormones, especially estrogens [27, 28].

Similarly, this prevalence of hypertension of $20.5 \%$ is also lower than $54.8 \%$ obtained by dos Prazeres et al. [9] in Angolan non diabetic pregnant women. This could be attributed to a high prevalence of obesity and characteristics of the study population of dos Prazeres and collaborators.

The prevalence of hyperglycaemia $(47.5 \%)$ in this study was higher than $13.3 \%$ obtained by dos Prazeres et al. [9] in non-diabetic Angolan pregnant women. The higher prevalence could be explained by the difference of cut-off values used. Hyperglycaemia was defined in the present study as fasting blood glucose $\geq 1.01 \mathrm{~g} / \mathrm{L}$ while Angolan study cut-off was $1.10 \mathrm{~g} / \mathrm{L}$.

The prevalence of low HDL (16.3\%), in the present study is higher than $2.5 \%$ reported by dos Prazeres et al. [9]. This inconsistency could be due to the cut-off value of HDL-C. Dos Prazeres et al. [9] in the study of metabolic syndrome in Angolan pregnant women, used the cut-off value of $0.40 \mathrm{~g} / \mathrm{L}$ while in the present study we had taken into consideration the variation of HDL-C during normal pregnancy and therefore, the later will probably decrease the prevalence of low-HDL$\mathrm{C}$ found in that study compared to the current study.

The prevalence of hypertriglyceridaemia of $3.7 \%$ found in the current study is in line with reports of Fezeu et al. [29] who also found that hypertriglyceridaemia was low or uncommon in Cameroonian adults. Similarly, this prevalence of hypertriglyceridaemia is almost similar to $2.2 \%$ reported by dos Prazeres et al [9], in non-diabetic Angolan pregnant women using the modified NCEP ATP III. This low prevalence of hypertriglyceridaemia could be partially due to the fact that: in the present study, only $0.12 \%$ of participants admitted to consume tobacco products and it has been proven that smoking tobacco products interfere with insulin function, leading to insulin resistance [30], increase in blood triglycerides levels, and reduction of HDL cholesterol [31]. Also the prevalence of hypertriglyceridaemia obtained in this study might have been influenced by alcohol consumption. About $60 \%$ of participants declared to be alcohol consumers, although, we could not assess the quality and quantity of alcohol taken by them. It has been observed that light to moderate alcohol consumption is associated with a lower prevalence of metabolic syndrome, with a favorable influence on lipids and fasting insulin [32].

The most common component of metabolic syndrome in the present study was hyperglycaemia, followed by obesity. This is inconsistent with findings reported by Ntentie et al. [8], who found that the most prevalent risk factor was abdominal obesity (49\%). This difference could be explained by the type of participants recruited and the fact that Ntentie et al. [8], used the waist circumference to define obesity while in the present study pre-pregnancy BMI was used.

\subsection{Distribution of Participants According the Number of Risk Factors of Metabolic Syndrome}

A majority of participants $(72.6 \%)$ had at least one risk factor of metabolic syndrome. These findings are in line with many studies that have demonstrated a high prevalence of non-communicable diseases and their risk factors in some sub-Saharan African settings [33].

\subsection{Comparison of Physiological and Biochemical Parameters of Participants with and Without Metabolic Syndrome}

Individuals with metabolic syndrome had significantly higher serum levels of triglycerides, fasting blood glucose 
and lower levels of HDL-C compared to individuals without metabolic syndrome. Also participants with metabolic syndrome had a higher value for DBP, SBP and BMI compared to those without it. These findings were consistent with the results reported by dos Prazeres et al. [9] in Angolan non diabetic pregnant women and also these parameters were involved in the definition of metabolic syndrome in the present study.

\subsection{Association Between Socio-Demographic \\ Characteristics and Metabolic Syndrome}

There were no significant association between metabolic syndrome and educational level in the present study. This contrasts findings by Hajian et al [34] who reported a higher prevalence of metabolic syndrome among participants with lower educational qualification. In fact, education has been documented to increase knowledge [35], and, it has been reported that more educated individuals are likely to be more receptive to new ideas, development and information provided regarding their health [36], thus education may affect lifestyle behaviour, psychosocial attitude, accessibility to health services, and economically advantageous surroundings.

The present study found no association between metabolic syndrome and marital status. This could be explained by the fact that in the questionnaire, we did not distinguish married participants who were living together and married participants who were living separately because of occupation duties. In fact, studies have shown that those who were married and lived together had better health behaviour than those who live alone [37], also the presence of a positive relationships provides physical and psychological support, especially providing a buffer in stressful situations [38, 39] which in turn reduce the occurrence of metabolic syndrome.

The absence of significant association between economic status and metabolic syndrome could be due to the definition of poverty used in the present study. In Cameroon, according to World Bank definition, a poor individual is an individual who spends less than 1.56 USD per day. In our definition we did not take into consideration the fact that in Cameroon, there are many individuals with insufficient financial income financially depending on a worker in the family. This psychological stress will lead to increase of level of cortisol which is a risk factor of hypertension [40, 41]. Also, this definition did not include the expensive cost of living in Douala and Yaoundé. Therefore the proportion classified as average economic situation was overestimated. On the contrary, it has been proven that increase in outcome has led to an increase in the purchasing capacity of the people and reluctance to do any manual work leading to an increase in the metabolic syndrome components.

Although cultural groups provide guidelines regarding acceptable foods, foods combinations, eating patterns, there was no significant association between ethnic group and metabolic syndrome in the present study. This could be explained by the fact that most of the participants were recruited in Douala and Yaoundé, which are the two main cosmopolitan towns in Cameroon.

Religious belief is known to influence or affect a follower's food choices and lifestyle. However, the present study did not show a significant association of religious status and metabolic syndrome. This could be explained by the fact that some "believer" did not really practice what their religion recommends.

\subsection{Association Between Metabolic Syndrome and Its Individual Components}

Fasting blood glucose and body mass index predicted most the risk of metabolic syndrome in the study population than other components. This could be justified by the high prevalence of metabolic these components in the current study. However, triglycerides and diastolic blood pressure did not associated with metabolic syndrome. This could be due explained in one part by the lower prevalence of hypertriglyceridemia found in the present study and lowest cut-off of high diastolic blood pressure used in this study.

\subsection{Association Between Medical History and Metabolic Syndrome}

Medical history in the present study included parity, gravidity, family (parent's) history of hypertension, type 2 diabetes mellitus and obesity.

About $27.1 \%$ of participants had parent's history of hypertension. This is in line with national prevalence of hypertension [26]. The prevalence of parent history of type 2 diabetes mellitus in the current study is higher than national prevalence which is $6 \%$. This could be probably due to the age of parent since prevalence of chronic diseases is known to increase with age.

\subsubsection{Correlation Between Parity, Gravidity and Components of Metabolic Syndrome}

The present study shows a fair, positive and significant correlation between parity and body mass index, and between gravidity and body mass index. This corroborates reports of previous studies [42, 43].

Also parity number was positively and significantly correlated to diastolic blood pressure $(r=0.09 ; p \approx 0.008)$. The results of the present study are in line with those of some previous studies, where higher parity was positively associated with blood pressure [44, 45].

Neither parity nor gravidity was significantly associated with other components of metabolic syndrome such as fasting blood glucose, HDL cholesterol, triglycerides. This is in line with reports of Akter et al [45] in Bangladesh rural women.

\subsubsection{Logistic Regression Analysis Between Medical History and Metabolic Syndrome}

Parity was positively associated with metabolic syndrome in the current study. This is in line with several studies reported worldwide. Indeed, repeated pregnancies lead to excess in weight gain or weight cycling and will lead to a greater upper fat distribution and higher prevalence of metabolic syndrome $[42,43,45,46]$. Also, normal pregnancy 
is a state of insulin resistance, and frequent pregnancies may result in permanent detrimental effects on lipids and glucose metabolisms [47, 48].

Gravidity was not significantly associated with metabolic syndrome. Previous studies have reported a higher risk of metabolic syndrome among participants with highest gravidity than those with lowest gravidity [42, 45]. This difference could be due to the duration of each pregnancy or its outcomes. However, information concerning the duration of previous pregnancies was not collected in the present study. Furthermore, questionnaire was self-reported and subjects may not have been aware of or correctly recall pregnancy losses or miscarriages. Also, live births are more likely to be recalled accurately than pregnancies.

Parent's positive history of hypertension was not significantly associated with metabolic syndrome in the present study. This is not consistent with a Sri Lankan study which reported significant association between family history of hypertension and metabolic syndrome [49]. This could be due to the different cut-off value of hypertension used in the both studies.

Participants with parent's history of type 2 diabetes mellitus had increased risk of developing metabolic syndrome than those with no history of type 2 diabetes mellitus. This is in line from previous studies [50].

\section{Strengths and Limitations of the Study}

This study addressed an important aspect of women's reproductive health which had been relatively underinvestigated in Cameroonian pregnant women. The survey data and measurements were collected first hand, and this contributed to completeness of data. Moreover, this study includes the estimation of the prevalence of metabolic syndrome components among pregnant women in the Centre and Littoral regions of Cameroon, making it is necessary to implement effective preventive measures.

However, this study had some limitations: Firstly: this study was carried out in two regions in Cameroon, the results are therefore difficult to generalize to the entire Cameroonian pregnant women. Secondly, there is no consensus criterion for obesity diagnosis during pregnancy, so we used the standard BMI at pre-pregnancy (self-reported) or BMI at the first quarter of pregnancy which can slightly affect our results. Thirdly, measurement errors as the result of selfreporting for socio-demographic and economic status may introduce non differentiation and misclassification leading to the association toward the null or less significance. Finally, the use of One Touch Ultra glucose meter (Point Of Care) for the determination of blood glucose could slightly affect ours results especially at low glucose concentration.

\section{Conclusion}

Metabolic syndrome is present among pregnant women in Cameroon with high prevalence of $7.0 \%$. More than twothird of participants had at least one risk factor of metabolic syndrome with hyperglycaemia and obesity being the most prevalent. Participants with metabolic syndrome had higher and significant values of FBG, BMI, TG, SBP and DBP than those without metabolic syndrome. High parity and parent history of type 2 diabetes mellitus were positively associated with a risk of metabolic syndrome in this study. These findings suggest that individuals with a parent's history of type 2 diabetes mellitus and multiparous pregnant women form an easily identifiable group who may benefit from targeted interventions. The high prevalence of metabolic syndrome, hyperglycemia, obesity and hypertension demonstrate the need to closely follow up pregnant women in order to minimize the risk of metabolic syndrome, cardiovascular diseases, type 2 diabetes mellitus, maternal and foetal adverse outcomes. Also, the used of various metabolic syndrome definitions in pregnancy do not enable to make comparisons between different studies. Therefore, a unified or harmonized definition is needed.

\section{Authors' Contributions}

Study concept and design: JDD and BPT; acquisition of data: JDD; analysis and interpretation of data: JDD; drafting of the manuscript: JDD; critical revision of the manuscript: BPT, JCNA, WEO and TNN; statistical analysis: JDD; administrative and material support: JDD and JCNA and study supervision: BPT, JCNA, TNN WEO and JDD.

\section{Declaration of Conflicting Interest}

The authors declare that they have no competing interest.

\section{Acknowledgements}

We would like to thank the Chief Medical Officer of Delangue Health Centre, Dr Edwige ABOMO OBAMA for her moral encouragements.

\section{References}

[1] Alberti K, Zimmet P, Shaw J. Metabolic syndrome: a new world-wide definition: a consensus statement from the International Diabetes Federation. Diabet Med $J$ Br Diabet Assoc 2006; 23 (5): 469-80.

[2] Wilson PW, D'Agostino RB, Parise H, Sullivan L, Meigs JB. Metabolic syndrome as precursor of cardiovascular disease and type 2 diabetes mellitus. Circulation 2005; 112: 3066-72.

[3] O'Neill S, O'Driscoll L. metabolic syndrome: a closer look at the growing epidemic and it associated pathologies. Obes. Rev. 2015; 16 (1): 1-12.

[4] Desroches S and Lamarche B. The evolving definitions and increasing prevalence of the metabolic syndrome. Appl Physiol Nutr Metab 2007; 32 (1), 23-32.

[5] Kolovou G, Anagnostopoulou K, Salpea K, Mikhailidis D. The prevalence of metabolic syndrome in various populations. Am J Med Scie 2007; 333 (6), 362-71. 
[6] Keates AK, Mocumbi AO, Ntsekhe M, Sliwa K, Stewart S. Cardiovascular Diseases in Africa: Epidemiological Profile and Challenges Nat Rev Cardiol. 2017; 14: 273-93.

[7] Vernay M, Salanave B, de Peretti C, Druet C, Malon A, Deschamps V, et al. Metabolic syndrome and socioeconomic status in France: The French Nutrition and Health Survey (ENNS, 2006-2007). Int J Public Health 2013; 58: 855-64.

[8] Ntentie FR, Ngondi JL, Azantsa KB, Santy EV, Dimodi HT, Mbong A et al. Urbanization and Metabolic Syndrome in Cameroon: Alertness on Less Urbanised Areas. Endocrinol Metab Syndr 2014; 3: 137-44.

[9] dos Prazeres T, Débora CD, Abbade JF, Negrato CA, de Campos PA, Calderon IM, et al. Prevalence of metabolic syndrome in non-diabetic, pregnant Angolan women according to four diagnostic criteria and its effects on adverse perinatal outcomes. Diabetol Metab Syndr 2016; 8: 27.

[10] Gunderson EP, Lewis CE, Murtaugh MA, Quesenberry CP, West SD, Sidney S. Long-term plasma lipid changes associated with a first birth: the Coronary Artery Risk Development in Young Adults study. Am J Epidemiol. 2004; 159: 1028-39.

[11] Smith DE, Lewis CE, Caveny JL, Perkins LL, Burke GL. Longitudinal changes in adiposity associated with pregnancy. The CARDIA Study. Coronary Artery Risk Development in Young Adults Study. JAMA 1994; 271: 1747-51.

[12] Cameron AJ, Shaw JE, Zimmet PZ, The metabolic syndrome: prevalence in worldwide populations. Endocrinol Metab. Clin North Am 2004; 33: 351-75.

[13] World Health Organization. The WHO STEPS Surveillance Manual, PART 5: Steps instrument. Last updated January 27, 2017. Accessible http://www.who.int/chp/steps/manual/en/index.html.

[14] Allain CC, Poon LS, Chan CS, Richmond W, Fu PC. Enzymatic determination of total serum cholesterol. Clin Chem 1974; 20: 470-5.

[15] Trinder P. Triglyceride estimation by GPD-PAP method. Ann Clin Biochem 1969; 6: 24-7.

[16] Burstein M, Scholnick HR, Morfin R. Rapid method for the isolation of lipoproteins from human serum by precipitation with polyanions. J Lipid Res 1970; 11: 583-95.

[17] Friedewald WT, Levy RI, Fredrickson DS. Estimation of the concentration of low density lipoprotein cholesterol in plasma without use of the preparative ultracentrifuge. Clin Chem 1972; 18 (6): 499-502.

[18] Piechota W, Staszewski A. Reference ranges of lipids and apolipoproteins in pregnancy. Eur J Obstet Gynecol Reprod Biol. 1992; 45 (1): 27-35.

[19] Halle EG, Nsom JB, Bechem NN, Palle NG, Mangala FN, Njotang NF. Outcome of Pregnancy in Patients with Pre Pregnancy Obesity in Douala, Cameroon. IJTDH, 2018; 32 (3): 1-10.

[20] Institut National de la Statistiques. Rapport Quatrième Enquête Camerounaise Aupres Des Ménages (ECAM 4). Institut National de la Statistique. CMR-INS-ECAM4-V1. 2. 2014, Cameroun

[21] Mpendulo G, Mang'unyi. Exploring Relationships between Education Level and Unemployment. J Soc Sci. 2018; 7 (2);
86: 86-102.

[22] Worldatlas. Ethnic groups of Cameroon. Accessible at https:www.worldatlas.com/articles/etnic group of Cameroon. htlm. Retrieved on 15/12/19.

[23] Central Bureau of Census and Population Studies. Third General Census of Population and Housing 2005. CMR-2005PHC- v01-M-v6. 3-A-IPUMS. 2005, Cameroon 2005. Accessible at. https:www.statistics-cameroon.org. Retrieved online on $5^{\text {th }}$ January 2020.

[24] Lima M, Melo AS, Sena AS, Barros V and Amorim MM. Metabolic syndrome in pregnancy and postpartum: prevalence and associated factors. Rev Assoc Med Bras 2019; 65 (12): 1489-95.

[25] Kamadjeu RM, Edwards R, Atanga JS, Kiawi EC, Unwin N, Mbanya JC. Anthropometry measures and prevalence of obesity in the urban adult population of Cameroon: an update from the Cameroon Burden of Diabetes Baseline Survey. BMC Public Health 2006. 6: 228.

[26] Kingue S, Ndong NC, Menanga A, Fesuh B, Nouedoui C, Muna WF. "Prevalence and risk factors of hypertension in urban areas of Cameroon: a nationwide populationbased cross-sectional study". J Clin Hyper 2015; 17 (10): 819-24.

[27] Hilliard LM, Sampson AK, Brown RD, Denton KM. The "his and hers" of the rennin -angiotensin system. Curr Hypertens Rep 2013; 15: 71-79.

[28] Xue B, Johnson AK, Hay M. Sex differences in angiotensin II and aldosterone-induced hypertension: the central protective effect of estrogen. Am J Physiol Regul Integr Comp Physiol 2013. Sep 1; 305 (5): R459-R463.

[29] Fezeu L, Balkau B, Kengne A, Sobngwi E, Mbanya JC. Metabolic syndrome in a Sub-Saharan Africa setting: Central obesity may be the key determinant. Atherosclerosis 2007; 193: $70-6$

[30] Attvall S, Fowelin J, Lager I, von Schenck H, Smith U. Smoking induces insulin resistance: a potential link with the insulin resistance syndrome. J Intern Med 1993; 233: 327-32.

[31] Kong C, Nimmo L, Elatrozy T, Anyaoku V, Hughes C, Robinson S, et al. Smoking is associated with increased hepatic lipase activity, insulin resistance, dyslipidaemia and early atherosclerosis in Type 2 diabetes. Atherosclerosis 2001; 156: $373-8$.

[32] Vieira BA, Luft VC, Schmidt MI, Chambless LE, Chor D, Barreto SM, et al. PLoS One 2016; 11 (9): e0163044.

[33] Yuyun MF, Sliwa K, Kengne AP, Mocumbi AO, Bukhman G. Cardiovascular Diseases in Sub-Saharan Africa Compared to High-Income Countries: An Epidemiological Perspective. Global Heart. 2020; 15 (1): 15.

[34] Hajian TK, Heidari B, Firouzjahi A, Bagherzadeh M, HajianTA, Halalkhor S. Prevalence of metabolic syndrome and the association with socio-demographic characteristics and physical activity in urban population of Iranian adults: a population based study. Diabetes Metab Syndr 2014; 8 (3): $170-6$.

[35] Cutler DM, Lleras MA. Understanding differences in health behaviors by education. J Health Econ 2010; 29 (1): $1-28$. 
[36] Deaton A, Cutler D, Lieras-Muney A. The determinants to mortality. J Econ Perspect 2006; 20 (3): 97-120.

[37] Schoenborn CA. Marital status and health; United State 19992002. US Department of Health and Human Services, Center for Diseases Control and Prevention. National Center for Health Statistic. 2004- Advance Data from vital and Health Statistic No 351 Dec 2004.

[38] Troxel WM, Matthews KA, Gallo LC, Kuller LH. Marital quality and occurrence of the metabolic syndrome in women. Arch Intern Med 2005; 165: 1022-7.

[39] Wyke S, Ford G. Competing explanations for associations between marital status and health. Soc Sci Med 1992; 34: 52332.

[40] Cohen S, Doyle WJ, Baum A. Socioeconomic status is associated with stress hormones. Psychosom Med 2006; 68 (3): 414-20.

[41] Whitworth JA, Mangos GJ, Kelly JJ. Cushing, cortisol and cardiovascular diseases. Hypertension 2000; 36 (5): 912-6.

[42] Després JP, Lemieux I. Abdominal obesity and metabolic syndrome. Nature 2006; 444: 881-7.

[43] Gunderson EP, Jacobs DR, Chiang V, Lewis CE, Tsai A, Quesenberry CP et al. Childbearing is associated with higher incidence of the metabolic syndrome among women of reproductive age controlling for measurements before pregnancy: the CARDIA study. Am J Obstet Gynecol. 2009; 201: 177: e1-e9.
[44] Taylor JY, Sampson DA, Anderson CM, Caldwell D, Taylor AD. Effects of parity on blood pressure among West African Dogon women. Ethn Dis. 2012; 22: 360-366.

[45] Akter S, Jesmin S, Rahman MM, Islam MM, Khatun MT, Yamaguchi $\mathrm{N}$ et al. Higher Gravidity and Parity Are Associated with Increased Prevalence of Metabolic Syndrome among Rural Bangladeshi Women. PLoS ONE 2013; 8 (8): e68319.

[46] Wu J, Xu G, Shen L, Zhang Y, Song L, Yang S et al. Parity and Risk of Metabolic Syndrome Among Chinese Women. J Women's Health. 2015; 24 (7): 602-07.

[47] Lewis GF, Steiner G. Acute effects of insulin in the control of VLDL production in humans. Implications for the insulinresistant state. Diabetes Care 1996; 19 (4): 390-3.

[48] Kritz-Silverstein D, Barrett-Connor E, Wingard D The relationship between multiparity and lipoprotein levels in older women. J Clin Epidemiol 1992; 45: 761-7.

[49] Ranasinghe P, Cooray DN, Jayawardena R, Katulanda P. The influence of family history of Hypertension on disease prevalence and associated metabolic risk factors among Sri Lankan adults. BMC Public Health. 2015; 15: 576.

[50] Das M, Pal S, Ghosh A. Family history of type 2 diabetes and prevalence of metabolic syndrome in adult Asian Indians. $J$ Cardiovasc Dis Res 2012; 3: 104-8. 